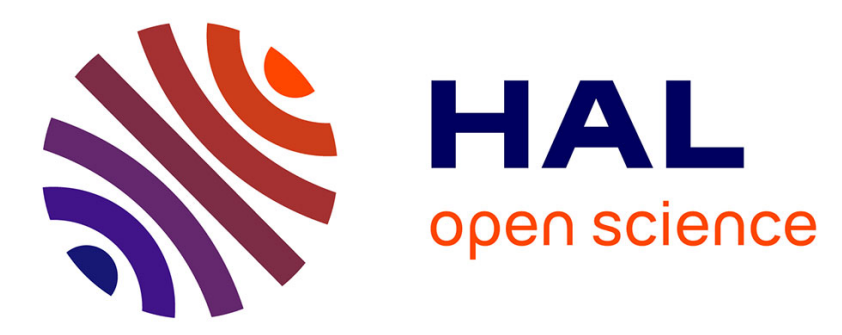

\title{
Présentation des possibilités de la diffraction de surface en ultra-vide sur les lignes françaises à l'ESRF
}

\author{
A. Barbier, R. Baudoing-Savois, G. Renaud, M. de Santis, O. Robach, \\ Marie-Claire Saint-Lager, P. Jeantet, P. Taunier, J.-P. Roux, O. Ulrich, et al.
}

\section{- To cite this version:}

A. Barbier, R. Baudoing-Savois, G. Renaud, M. de Santis, O. Robach, et al.. Présentation des possibilités de la diffraction de surface en ultra-vide sur les lignes françaises à l'ESRF. Journal de Physique IV Proceedings, 1996, 06 (C4), pp.C4-341-C4-349. 10.1051/jp4:1996431 • jpa-00254314

\section{HAL Id: jpa-00254314 https://hal.science/jpa-00254314}

Submitted on 1 Jan 1996

HAL is a multi-disciplinary open access archive for the deposit and dissemination of scientific research documents, whether they are published or not. The documents may come from teaching and research institutions in France or abroad, or from public or private research centers.
L'archive ouverte pluridisciplinaire HAL, est destinée au dépôt et à la diffusion de documents scientifiques de niveau recherche, publiés ou non, émanant des établissements d'enseignement et de recherche français ou étrangers, des laboratoires publics ou privés. 


\title{
Présentation des possibilités de la diffraction de surface en ultranvide sur les lignes françaises à l'ESRF
}

\author{
A. Barbier, R. Baudoing-Savois*, G. Renaud, M. de Santis*, O. Robach, M.-C. Saint-Lager*, \\ P. Jeantet**, P. Taunier*, J.-P. Roux**, O. Ulrich, A. Mougin, G. Berard et O. Geaymond* \\ Département de Recherche Fondamentale sur la Matière Condensée SP2M/PI, CEA/Grenoble, \\ 17 rue des Martyrs, 38054 Grenoble cedex, France \\ * CNRS, Laboratoire de Cristallographie, BP. 166, 38042 Grenoble cedex, France \\ ** CNRS-SERAS, BP. 166, 38042 Grenoble, France
}

\begin{abstract}
Résumé : Après une brève introduction portant sur l'étude de surfaces et d'interfaces par rayons $X$ nous présentons dans cet article un équipement nouvellement construit à l'ESRF (European Synchrotron Radiation Facility) sur la ligne D32(CRG-IF IF i.e. Collaborating Reseach Group - Interface). Il s'agit d'un goniomètre équipé d'une chambre ultra-vide ainsi que d'un système de transfert et d'introduction rapide d'échantillon; il permet de réaliser des études par diffraction de rayons $\mathrm{X}$ en incidence rasante de surfaces et d'interfaces in situ. Nous décrivons brièvement les appareils antérieurs en précisant quelles sont les améliorations apportées par notre équipement sans toutefois entrer dans des détails trop techniques. De premiers résultats sont aussi présentés.
\end{abstract}

\begin{abstract}
After a brief introduction to the matter of X-rays diffraction at surfaces and interfaces we present in this paper an original and new equipment recently built at the ESRF (European Synchrotron Radiation Facility) on beamline D32(CRG-IF i.e. Collaborating Reseach Group - Interface). This goniometer is equipped with an ultrahigh vacuum chamber, a sample transfer and an introduction system. It allows one to study the growth via grazing incidence $\mathrm{X}$ rays diffraction of in situ prepared surfaces and interfaces. Without giving all technical details we also discuss the improvement we have made on this apparatus in regard to previous ones. First results are also presented.
\end{abstract}

\section{INTRODUCTION}

De nombreuses applications technologiques reposent actuellement sur les propriétés siégeant au niveau des surfaces et des interfaces de matériaux artificiels. L'intérêt pour ce type d'études est grandissant depuis plusieurs décennies que ce soit du point de vue fondamental ou appliqué [1]. Les comportements électroniques et magnétiques, tout à fait originaux, des atomes dans ces sites particuliers permettent d'en tirer profit pour imaginer de nouvelles structures. Il est évident que la configuration géométrique dans lesquelles se trouvent ces atomes a une importance capitale. Toute une variété de techniques a été mise au point pour l'étude de telles structures, chacune avec ses avantages et ses inconvénients. La diffraction d'électrons lents a longtemps dominée les études structurales; c'est une technique facile à mettre en oeuvre expérimentalement mais qui d'un autre coté impose le traitement de la diffusion multiple des électrons. Plus récemment la microscopie par effet tunnel a fait son apparition, elle donne une vision des atomes en surface mais elle n'a pas accès aux interfaces. Ces deux méthodes sont tout à fait inadaptées à l'étude de matériaux isolants et des interfaces enterrées.

La diffraction des rayons $X$ a, quant à elle, donné toute satisfaction en tant que méthode d'analyse des matériaux massifs, et nombre de structures complexes ont pu être déterminées précisément parce qu'un traitement cinématique est suffisant dans ce cas et qu'elle n'est pas 
limitée aux échantillons non isolants. Malgré quelques fort belles études réalisées avec des anodes tournantes sur des éléments lourds tels que l'or[2], la principale raison qui gênait l'extension de cette puissante méthode à l'étude des surfaces et des interfaces était la faiblesse de l'intensité diffractée par celles-ci. Pour des éléments plus légers l'intensité lumineuse obtenue à partir d'une anode tournante est inférieure de 2 à 3 ordres de grandeurs au minimum nécessaire pour étudier les reconstructions de ces matériaux. De plus seule une fraction de l'échantillon de l'ordre de $1 \times 0.1 \mathrm{~mm}^{2}$ est analysée, on aura donc tout intérêt à disposer d'une source de photons bien focalisée. Ce n'est donc qu'en 1979 que Marra, Eisenberger et Cho[3] ont montrés que la diffraction de surface à partir de couches atomiques d'Al sur GaAs était possible et que la sensibilité à la surface était grandement favorisée si l'angle d'incidence des rayons X est faible. En 1981[4] une première étude de reconstruction de surface en ultra-vide a été menée par diffraction de surface à la fois avec une anode tournante et par rayonnement synchrotron. Cette approche ainsi que les appareillages n'ont cessé de se développer depuis. La diffraction due à quelques atomes sur une surface peut à présent être observée avec des sources de troisième génération, tel l'ESRF (European Synchrotron Radiation Facility) implanté à Grenoble (France).

La communauté française CEA/CNRS s'est dotée de deux lignes de lumière sur des aimants de courbure de cet anneau de stockage. Sur la ligne CRG-IF un goniomètre ultra-vide (SUV) permettant de telles études de surfaces et d'interfaces vient d'être installé.

Tout en portant l'accent sur les possibilités d'études accessibles à partir de cet instrument cet article se décomposera ainsi : Dans la section 2 nous décrirons brièvement la ligne de lumière CRG-IF. Nous rappellerons les principes de la diffraction des rayons $X$ en incidence rasante appliquée à l'étude des surfaces ainsi que le choix de géométrie que nous avons fait dans la troisième partie. Le goniomètre ainsi que ses différents éléments sont décrit dans la quatrième partie, un exemple illustratif d'étude dans la cinquième.

\section{LA LIGNE DE LUMIERE}

Différents pays européens, dont la France, ont acquis des lignes de lumière sur les aimants de courbures à l'ESRF. La ligne CRG-IF est l'une d'entre elles.

Cette ligne comporte une cabane optique et trois stations expérimentales : la première permet des analyses de XAS (X-Ray absorption Spectroscopy), la seconde comporte un goniomètre multitechniques et enfin la dernière, prèsentée ici, est dédiée à l'étude de la diffraction en incidence rasante dans un environnement ultravide.

L'optique est constituée d'un miroir de conception originale et d'un monochromateur :

* Le miroir est recouvert d'une bande de Pt et d'une bande de Ni qui peuvent être mises alternativement dans le faisceau assurant ainsi la réjection des harmoniques à 20 et $30 \mathrm{KeV}$ respectivement pour un angle par rapport au faisceau valant $2.9 \mathrm{mrad}$. D'autre part il assure aussi la focalisation verticale, son rayon de courbure pouvant être varié continument de 6.5 à $35 \mathrm{~km}$. Il est possible de collimater ou de focaliser le faisceau dans chacune des 3 stations expérimentales.

* Le monochromateur dispose de 2 cristaux de Si(111) dont le deuxième peut être courbé continument pour assurer la focalisation sagittale ou la collimation dans chacune des trois stations expérimentales. L'énergie est ajustable entre 4 et $30 \mathrm{KeV}$. Sa résolution en énergie $\Delta \mathrm{E} / \mathrm{E}$ est de l'ordre de $2.10^{-4}$.

La taille du faisceau focalisé dans l'expérience SUV est de $0.25 \times 0.3 \mathrm{~mm}^{2}(\mathrm{H} \times \mathrm{V})$ avec un flux de l'ordre de $10^{11} \mathrm{ph} \mathrm{s}^{-1}(0.01 \% \text { bw, } 0.1 \mathrm{~A} \text { à } 20 \mathrm{KeV})^{-1}$. 


\section{DIFFRACTION DE RAYONS X EN INCIDENCE RASANTE - GEOMETREE}

\subsection{La diffraction de rayons $X$ en incidence rasante}

Dans cette partie nous rappelons les principes élémentaires qui justifient l'étude des surfaces en incidence rasante. Des descriptions beaucoup plus détaillées pourront être trouvées dans d'excellents articles de revue assez récents qui traitent ce sujet plus complètement [5-6].

La propriété fondamentale qui est exploitée ici est la réflexion totale de la lumière par une surface. Celle-ci, tout comme pour la lumière visible, est gouvernée par l'indice de réfraction $\mathbf{n}$ qui s'écrit pour un matériau ordonné :

$$
\begin{aligned}
& \mathrm{n}=1-\delta-\mathrm{i} . \beta \\
& \text { où : } \delta=\frac{\lambda^{2} \mathrm{e}^{2}}{2 \pi m \mathrm{c}^{2} v} \sum_{\text {maille }}\left(Z_{\mathrm{j}}+f_{\mathrm{j}}^{\prime}\right) \text { et } \beta=\frac{\lambda^{2} \mathrm{e}^{2}}{2 \pi m \mathrm{c}^{2} v} \sum_{\text {maille }} f_{\mathrm{j}}^{\prime \prime}=\frac{\lambda \mu}{4 \pi}
\end{aligned}
$$

Où $\lambda$ est la longueur d'onde, c la vitesse de la lumière, $m$ et e la masse et la charge de l'électron, $v$ le volume de la maille, $Z_{\mathrm{j}}$ les charges atomiques, $f$ ' et $f$ " les corrections de dispersion des facteurs de diffusion atomiques et $\mu$ le coefficient d'absorption linéaire.

Pour les rayons $X$, n est légèrement inférieur à 1 car $\delta$ et $\beta$ sont de l'ordre de $10^{-5}$ et $10^{-6}$ respectivement. Les rayons $X$ ne seront donc réfléchis totalement que pour des angles d'incidence $\alpha_{i}$ trés petits et inférieurs à l'angle critique : $\alpha_{c}-\sqrt{2 \delta} \sim 0.1-0.5^{\circ}$ (pour des longueurs d'onde de l'ordre de $1.5 \AA$ ). Pour $\alpha_{i} \leq \alpha_{c}$ un champ d'ondes stationnaires s'installe entre faisceau incident et faisceau réfléchi; le faisceau transmis ne se propage plus perpendiculairement à la surface et son amplitude est doublée (fig. 1a). Pour des angles d'émergence $\alpha_{1} \leq \alpha_{c}$ les mêmes effets de réfraction se produisent. La figure 1b montre que dans de telles conditions géométriques $\left(\alpha_{i} \leq \alpha_{c}\right.$ et $\left.\alpha_{f} \leq \alpha_{c}\right)$ la profondeur de pénétration $\Lambda$ des rayons $X$ peut être très limitée (quelques dizaines d'Angstroems).
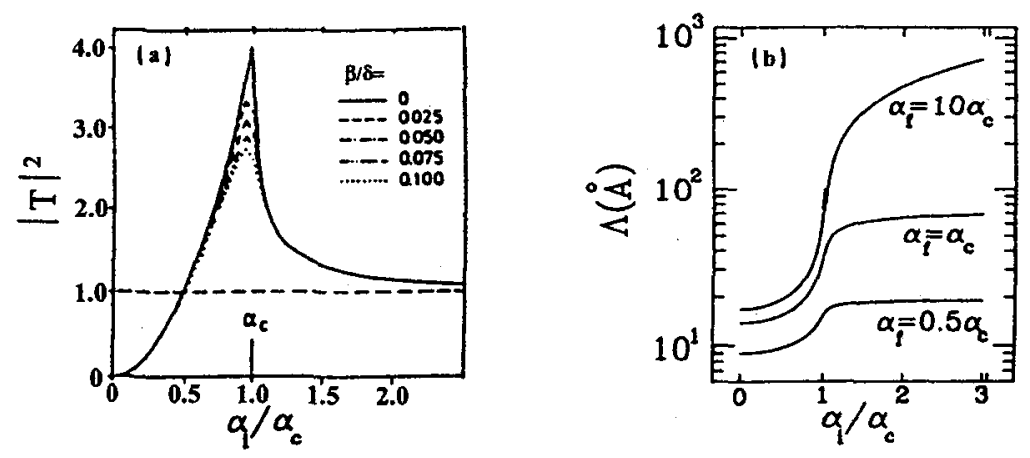

Figure 1 : (a) Facteur de transmission $|T|^{2}$ des rayons $X$ en fonction de $\alpha_{i}$ pour différents $\beta / \delta$ (b) Variation de la profondeur de pénétration des rayons $\mathrm{X}$, de longueur d'onde $1.5 \AA$, en fonction de l'angle d'incidence $\alpha_{i}$ et de l'angle d'émergence $\alpha_{f}$ pour une surface de $P t$. - (a) X-ray transmissivity $|\mathrm{T}|^{2}$ versus $\alpha_{i}$ for various $\beta / \delta$ (b) Scattering depth versus incident $\alpha_{i}$ and exit $\alpha_{i}$ angles for a Pt surface and a $1.5 \AA$ wavelength.

L'étude des interfaces peut se faire en augmentant $\alpha_{i}$. Pour des angles d'incidence croissants et supérieurs à $\alpha_{c}$ les rayons $X$ pénètrent de plus en plus profondément dans le matériau et l'analyse de l'intensité diffractée fournit alors des informations de manière non 
destructive sur les interfaces enterrées. L'angle d'incidence $\alpha_{i}$ est d'une importance capitale pour ces expériences, il devra donc pouvoir être défini précisément.

Dans le cas de surfaces et d'interfaces les conditions de diffraction sont relaxées. Le réseau réciproque qui en découle est un réseau constitué de pic de Bragg et de tiges d'intensité mesurable qui relient ces pics dans la direction perpendiculaire à la surface. De plus l'intensité diffusée aux minima de ces tiges par une surface est de l'ordre de celle qui serait diffusée par une demi-couche du même matériau. En ce point les déphasages entre plans voisins du volume parallèlement à la surface annulent l'essentiel de la contribution de volume ; on aura donc une très grande sensibilité à la surface d'une part mais aussi une impressionnante sensibilité à des atomes adsorbés. Encore faut-il pouvoir mesurer ces tiges, c'est à dire se déplacer dans l'espace réciproque sans changer l'angle $\alpha_{i}$. De telles mesures imposent donc que les mouvements du détecteur ne soient pas tributaires du mouvement $\alpha_{j}$.

D'un point de vue pratique il faudra de plus que l'échantillon possède une rotation autour de la normale à sa surface. De plus il faut que le faisceau soit bien défini avant l'échantillon et avant le détecteur. Sous ces contraintes la géométrie générale d'une expérience de diffraction sera celle donnée dans la figure 2 .

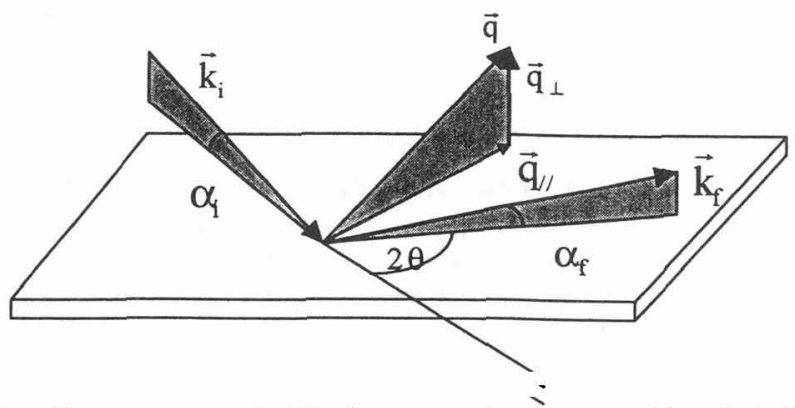

Figure 2 : Geometne d'une expenence de dittraction en incidence rasante. $\left(k_{i}, \alpha_{i}\right)$ et $\left(k_{f}, \alpha_{j}\right)$ sont les vecteurs d'onde et angles incident et émergent respectivement. $2 \theta$ est l'angle de diffraction. Le transfert de moment $\bar{q}$ possède une composante dans le plan et une autre hors du plan. - Glancing angle geometry. $\left(\vec{k}_{i}, \alpha_{i}\right)$ and $\left(\vec{k}_{\mathrm{f}}, \alpha_{\mathrm{f}}\right)$ are the incident and emergent wave vectors and angles respectively. $2 \theta$ is the diffraction angle. The momentum transfer $\bar{q}$ is composed of an in-plane and a normal component.

Dans le cas de l'utilisation d'un rayonnement synchrotron comme source de lumière une dernière exigence géométrique liée à ce type d'expérience vient s'ajouter aux précédentes. Elle est associée à la spécificité du rayonnement lui-même. Les rayons $X$ issus d'un synchrotron sont fortement polarisés dans le plan de l'orbite des électrons, horizontal. Si l'échantillon était monté horizontalement la détection serait tributaire du facteur de polarisation $P=\cos ^{2} 2 \theta$ ce qui interdirait toute mesure à $90^{\circ}$. Pour des études cristallographiques l'échantillon sera donc monté verticalement, le facteur de polarisation sera alors unitaire pour des mesures dans le plan. Une telle disposition présente un autre avantage : pour des lignes de lumière doublement focalisées comme la notre (Cf. §2) la divergence horizontale est largement supérieure à la divergence verticale. La fonction de résolution sera étalée selon la normale à l'échantillon, donc parallèlement aux tiges de diffraction, maximisant ainsi leurs intensités. Cependant pour des expériences de spectroscopie de type SEXAFS en détection de fluorescence ou de reflectivité il sera avantageux d'être en géométrie horizontale. 


\subsection{Choix de la géométrie pour l'instrument SUV}

L'étude de surfaces réactives ou d'interfaces en cours de croissance impose au-delà des conditions géométriques du $\S 3.1$ des possibilités de conditionnement et de caractérisation dans un environnement ultra-vide (UHV). On comprendra aisément que la conception d'un outil assujetti à autant de contraintes n'est a priori pas aisé.

Dans les premières expériences[7] de ce type, l'échantillon était préparé dans un équipement UHV standard puis transféré dans une petite chambre sous vide placée sur des goniomètres 2 ou 4 cercles. La chambre annexe possédait une fenêtre de béryllium permettant le passage des rayons $\mathrm{X}$. La durée de mise en oeuvre était très longue et ne permettait pas de caractériser l'échantillon pendant les expériences de rayons $X$. Par conséquent une telle approche était restreinte à l'étude de surface assez inertes.

Pour palier ces inconvénients, des diffractomètres plus complets furent construits[8]. Ils possédaient de larges translations selon $Z$ (dans la direction de la normale à la surface) et la chambre UHV tournait sur elle-même par accouplement au goniomètre, imposant ainsi une fenêtre en $\mathrm{Be}$ de $360^{\circ}$. Dans un tel dispositif peu d'outils pouvaient être montés car le poids total devait rester limité. D'autre part la rotation n'était guère précise à cause du poids supporté.

L'amélioration suivante[9] consista à ne faire bouger l'ensemble de l'équipement UHV que modérément pour définir l'angle d'incidence, mais de transmettre la rotation de l'échantillon autour de sa normale à partir du goniomètre par l'intermédiaire d'un cercle à pompage différentiel. Ainsi la chambre reste fixe et de larges possibilités d'équipement sont alors envisageables nonobstant le poids total pour peu que le centre de gravité soit placé sur l'axe de rotation du cercle définissant l'angle d'incidence. Plusieurs diffractomètres ont été construits d'après ces principes[10-11]

Dans une réalisation plus récente G.Renaud et coll.[12] ont construit un diffractomètre pour lequel les cercles $\chi$ et $\phi$ des goniomètres 4 cercles ont été remplacés par deux berceaux $\chi_{1}$ et $\chi_{2}$ d'extension limitée. Un tel montage permet de réaliser des gains d'encombrement et de précision indéniables. Pour l'obtention des géométries de surface verticale et horizontale deux petites chambres UHV étaient montables sur le goniomètre. Avec cet équipement on ne pouvait pas passer d'un type d'expérience à l'autre sans changer la chambre UHV.

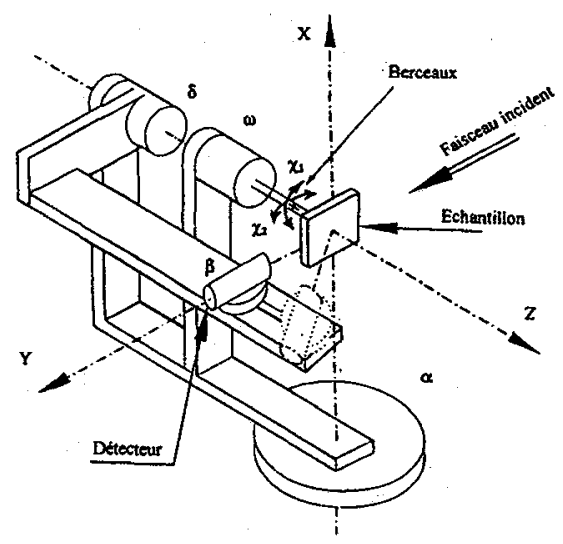

Figure 3 : Schéma de principe des mouvements sur le diffractomètre SUV. - Schematic representation of the goniometer's movements. 
L'équipement que nous venons de construire (SUV) est destiné à pouvoir réaliser un grand • nombre d'expériences différentes autant en géométrie verticale (diffraction de surface) qu'en géométrie horizontale (ReflSEXAFS et réflectométrie). Il est fondé sur la demière approche, mais dans notre cas la chambre UHV est bien plus importante car elle n'a pas à être démontée pour passer d'une géométrie à l'autre. C'est l'embout porte échantillon lui-même qui peut pivoter de $90^{\circ}$. Ainsi l'ensemble des études envisageables peut être réalisé sur le même échantillon.

Compte tenu des contraintes et des expériences envisagées, la géométrie du diffractomètre SUV a été choisie suivant le schéma de principe représenté sur la figure 3. Les mouvements $\alpha$ (angle d'incidence fig.4-3), $\beta$ (angle d'émergence fig4-18 et 11), $\delta$ (angle de rotation du détecteur autour de la normale à l'échantillon fig.4-10) et $\omega$ (angle de rotation de l'échantillon autour de la normale à sa surface fig.4-8, transmis à l'échantillon par un passage à pompage différentiel) sont supportés par le goniomètre alors que $\chi_{1}, \chi_{2}, \mathrm{X}$ et $\mathrm{Z}$ sont propres au manipulateur.

\section{DESCRIPTION DE L'EXPERIENCE SUV}

Le goniomètre 4 cercles a été construit par GMI (Grenoble) et la tête porte échantillon a été conçue et réalisée par le SERAS (Service d'Etudes et de Réalisations d'Appareillages Scientifiques du CNRS à Grenoble). L'angle $\beta$ d'orientation du détecteur est obtenu par la combinaison d'un mouvement de translation $\beta$ trans (fig.4-18) et d'un mouvement de rotation $\beta r o t$ (fig.4-11). Les axes $\delta$ et $\omega$ ont été alignés à l'horizontale à mieux que $0.003^{\circ}$, l'axe $\alpha$ est vertical à mieux que $0.002^{\circ}$. La sphère de confusion de l'ensemble des cercles du goniomètre est de l'ordre de $35 \mu \mathrm{m}$. En plus des différents degrés de liberté déjà évoquées, la hauteur $\mathbf{H}$ (fig.4-2) et la position latérale $\mathrm{T}$ (fig.4-1) du goniomètre sont pilotées. Le tableau I récapitule les caractéristiques des différents mouvements possibles.

TABLEAU I : Caractéristiques des cercles - The circles caracteristics

\begin{tabular}{|c|c|c|c|c|c|c|c|c|c|c|c|}
\hline \multirow[b]{2}{*}{ Cercle } & \multicolumn{5}{|c|}{ Goniomètre 4 cercles } & & & \multicolumn{4}{|c|}{ Tête goniométrique } \\
\hline & $\begin{array}{c}\alpha \\
(\mathrm{deg})\end{array}$ & $\begin{array}{c}\text { Brot } \\
\text { (deg) }\end{array}$ & $\begin{array}{l}\text { Btrans } \\
(\mathrm{mm})\end{array}$ & $\begin{array}{c}\delta \\
\text { (deg) }\end{array}$ & $\begin{array}{c}\omega \\
(\mathrm{deg})\end{array}$ & $\begin{array}{c}\mathrm{H} \\
(\mathrm{mm}) \\
\end{array}$ & $\begin{array}{c}\mathrm{T} \\
(\mathrm{mm})\end{array}$ & $\begin{array}{c}\chi_{1} \\
(\mathrm{deg})\end{array}$ & $\begin{array}{c}\chi_{2} \\
(\operatorname{deg}) \\
\end{array}$ & $\begin{array}{c}\mathrm{X} \\
(\mathrm{mm}) \\
\end{array}$ & $\begin{array}{c}\mathrm{Z} \\
(\mathrm{mm}) \\
\end{array}$ \\
\hline $\begin{array}{l}\text { Résolution mécanique } \\
\text { par pas de moteur }\end{array}$ & 0.0005 & 0.0005 & 0.005 & 0.001 & 0.0005 & 0.0005 & 0.0005 & & & & \\
\hline Déplacements $\max$ & 45 & 45 & 500 & 135 & \pm 200 & 50 & 50 & \pm 4 & \pm 4 & \pm 5 & \pm 5 \\
\hline Faux rond $(\mu \mathrm{m})$ & $\leq \pm 1$ & $<2$ & & $<2$ & $< \pm 1$ & & & & & & \\
\hline Voilage $(\mu \mathrm{m})$ & $<12$ & 2 & & $<2$ & $< \pm 3$ & & & & & & \\
\hline Précision & $<0.01$ & $<0.01$ & & $<0.01$ & $<0.01$ & & & & & & \\
\hline Concentricité $(\mu \mathrm{m})$ & $\leq 30$ & & & $\leq 30$ & $\leq 20$ & & & & & & \\
\hline Répétabilité & $\leq 0.005$ & $\leq 0.001$ & & $\leq 0.01$ & $\leq 0.005$ & & & 0.01 & 0.01 & 0.005 & 0.005 \\
\hline
\end{tabular}

Le faisceau de rayons $X$ est défini avant l'échantillon par un ensemble de 2 paires de fentes $(\mathrm{H} \times \mathrm{V})$ symétriques, réalisées par la société Micro-Controle, distantes de $1 \mathrm{~m} 80$. Elles sont motorisées en translation $( \pm 5 \mathrm{~mm} ; 0.13 \mu \mathrm{m}$ de résolution) et en ouverture $(0 \mathrm{à} 10 \mathrm{~mm} ; 0.3 \mu \mathrm{m}$ de résolution). Elles sont montées dans un rail de type X95 et sous vide primaire pour limiter la diffusion par l'air. 


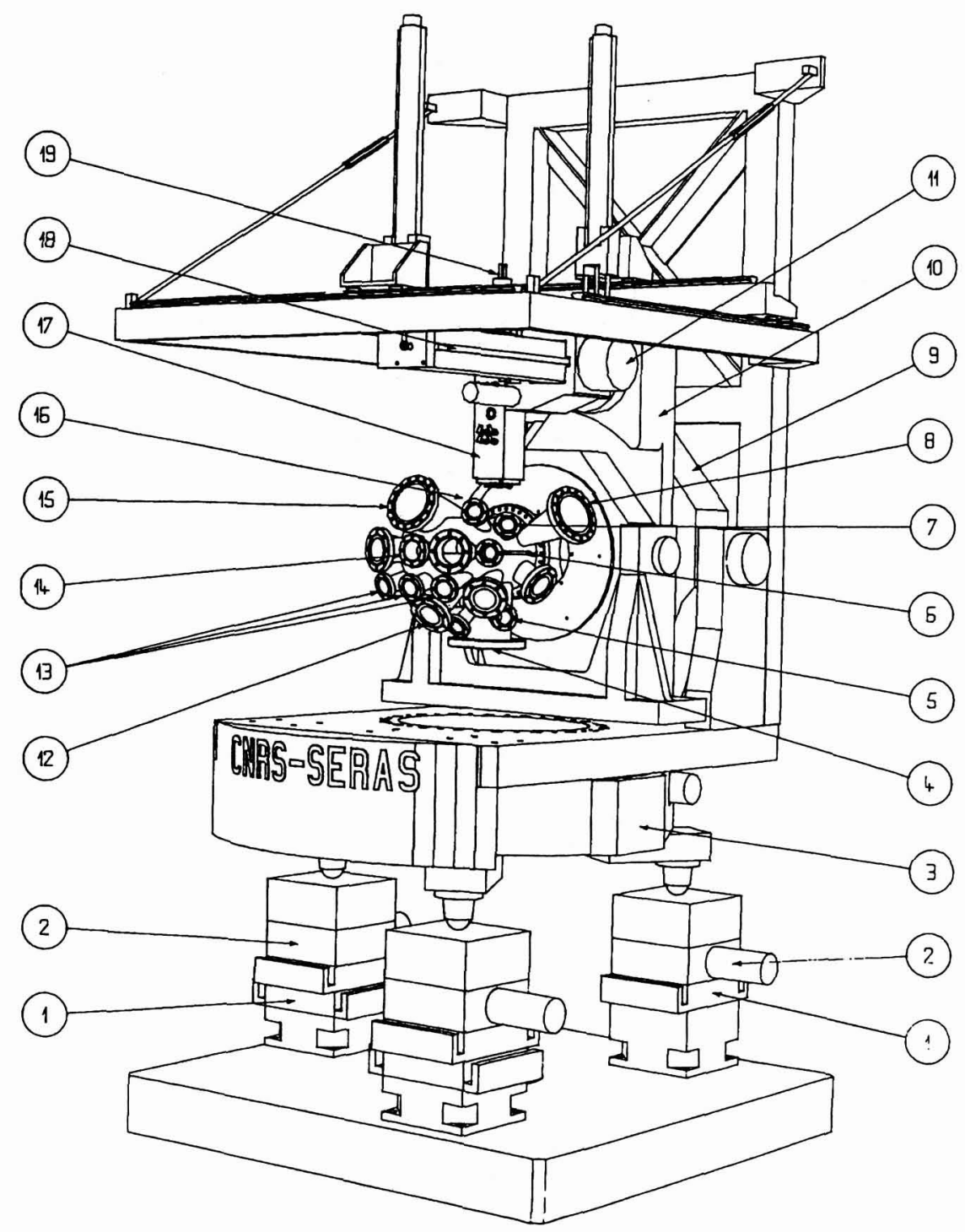

Figure 4 : Représentation schématique du goniomètre UHV. Par mesure de clareté les instruments et le pompage ne sont pas représentés - Schematic représentation of the UHV goniometer, for simplicity only the chamber is represented without equipments and pumping units.

La résolution angulaire pour la détection est assurée par deux autres couples de fentes (fig.4-17) motorisées placées elles aussi dans un rail X95, sous vide primaire statique et distantes de $30 \mathrm{~cm}$. La motorisation de toutes les fentes définissant le faisceau est tout à fait originale et permet une grande maniabilité. 
Les rayons $X$ pénètrent dans la chambre UHV par une petite fenêtre en béryllium (fig.4-6), les faisceaux diffractées sortent de la chambre par une plus grande fenêtre (fig.4-16). La chambre est par ailleurs équipée d'outils de préparation et de caractérisation classiques (figure 4) : Canon et écran RHEED associé à une imagerie informatisée, analyseur Auger de type CMA(fig.4-15), une balance à quartz, 4 sources d'épitaxie par jets moléculaires (fig.4-13), un quadrupole pour l'analyse des gaz résiduels ainsi que d'un système de pompage (fig.4-4) composé d'une pompe turbo principale, d'une petite pompe turbomoléculaire pour le cercle à pompage différentiel et d'une pompe ionique sous la chambre mais solidaire du cercle $\alpha$. La détection d'électrons pour la spectroscopie d'absorption X de surface (SEXAFS) est en cours d'implémentation. Le soufflet (fig.4-14) est utilisé pour les transferts d'échantillons qui sont montés sur des molyblocs de diamètre 1 pouce via un ensemble modutrack de RIBER. Dans ce modutrack les échantillons sont transférés sur des chariots mobiles, il pourront ainsi être amené vers d'autres éléments (chambre d'épitaxie, STM, chambres de préparations et de caractérisation annexes) de l'équipement qui se trouvent en dehors du rayonnement $\mathrm{X}$. Ces outils supplémentaires ne sont pas encore montés.

\section{EXEMPLE}

Les premières expériences nous ont permis de montrer que le goniomètre était opérationnel et que l'on peut reproduire des mesures de tiges de troncatures publiées dans la littérature. Nous présentons ici un seul exemple illustratif des capacités du goniomètre et de la méthode.

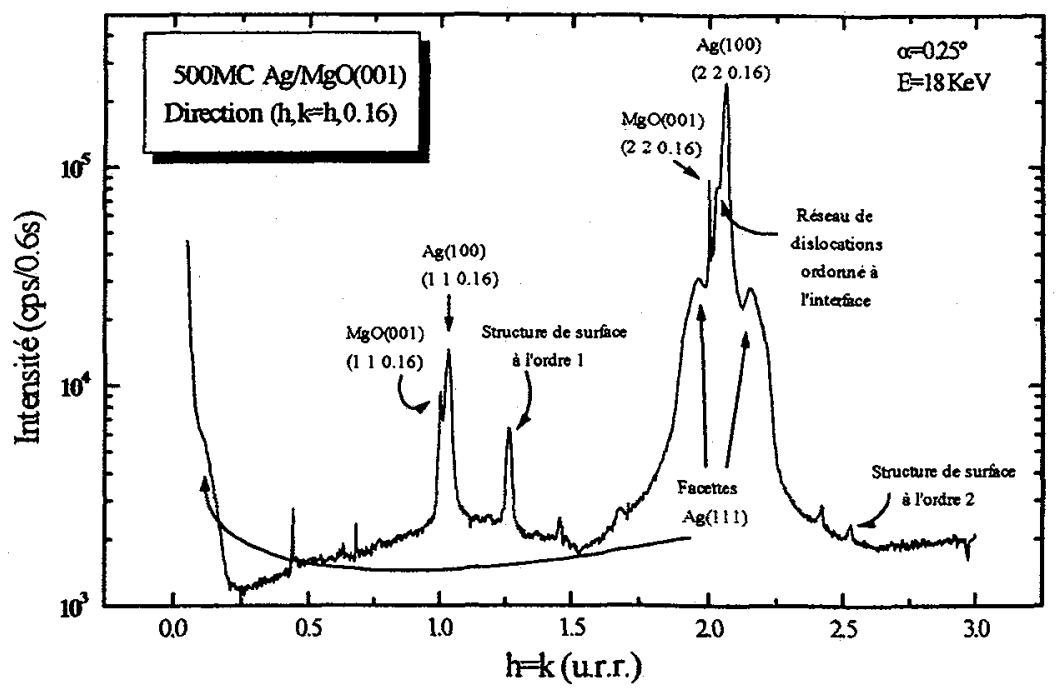

Figure 5 : Exemple d'une coupe transverse dans l'espace réciproque de 500 monocouches d'Ag sur $\mathrm{MgO}(001)$. - Example of a transverse scan in the reciprocal space of 500 monolayers $\mathrm{Ag}$ on $\mathrm{MgO}(00 \mathrm{I})$.

Il s'agit d'un enregistrement (fig. 5) de l'intensité diffractée (selon une coupe transverse $(h, k=h, l=0.16)$ en unité du réseau réciproque (u.r.r.)) par un monocristal de $\mathrm{MgO}(001)$ recouvert d'environ 500 couches d'Ag. L'angle d'incidence est de $0.25^{\circ}$ et l'énergie du rayonnement vaut $18 \mathrm{KeV}$. Nous voyons sur ce spectre des informations relatives à : (i) l'ordre dans le substrat (pics de Bragg (220) du MgO), (ii) la structure du film lui-même (pics de 
Bragg (220) de l'Ag relaxé) (iii) la structure de l'interface (pics de dislocations) (iv) facettes de l'Ag de type (111) (v) pics liés la structure de la surface (reconstruction ou alliage de surface), l'appartenance à la surface est mise en évidence par des mesures identiques pour des angle $S$ d'incidence différents et (vi) l'existence de tiges de troncatures (110) de l'Ag et (110) du MgO près des conditions anti-Bragg (la tige coupe le spectre de la figure 5 autour de $h=1$ ).

Ainsi les rayons $\mathrm{X}$ en incidence rasante permettent de sonder à la fois le substrat, l'interface, la morphologie du dépôt et sa surface.

\section{CONCLUSIONS ET PERSPECTIVES}

Dans cet article nous avons brièvement décrit un équipement nouvellement construit à l'ESRF pour réaliser des expériences de diffraction en incidence rasante. En mettant l'accent sur les principes qui ont conduit à la construction de ce goniomètre et en rappelant les bases de la méthode nous avons illustrés les capacités appréciables de cette instrument. Il devrait permettre de résoudre bon nombre de problèmes de surfaces et d'interfaces inaccessibles par d'autres méthodes.

Cependant cet ensemble n'est pas complet à l'heure actuelle. Un développement supplémentaire de la chambre UHV sera l'ajout d'un détecteur d'électrons pour réaliser des expériences de SEXAFS ; un détecteur en fluorescence est à l'étude. En dehors de la partie irradiée mais accessible par le système de transfert seront installés dans l'année à venir une chambre d'épitaxie par jets moléculaire, une chambre de caractérisation (LEED dynamique/Auger) et un STM. Cet ensemble expérimental sera alors sans nul doute tout à fait unique pour l'étude des surfaces et interfaces.

\section{Remerciements.}

Les auteurs tiennent à remercier l'ensemble des personnels techniques du SERAS et du laboratoire SP2M/PI pour leur disponibilité et leur assistance.

\section{Références}

[1] Surface Science " The first thirty years" (Edité par C.B.Duke, Elsevier science publishers B.V. 1994)

[2] I.K.Robinson,Phys. Rev. Lett. 50 (1983) 1145

[3] W.C. Marra, P.Eisenberger et A.Y.Cho, J. Appl. Phys.50 (1979) 6927

[4] P.Eisenberger et W.C.Marra, Phys. Rev. Lett. 46 (1981) 1081

[5] R. Feidenhans'1, Surf. Sci. Rep. 10 (1989) 105

[6] H.Dosch, Critical Phenomena at Surfaces and Interfaces, (Springer-Verlag, Tracts in Modern Physics Vol 126)

[7] R.L.Johnson, J.H.Fock et coll., The structure of surfaces (Springer-Verlag, Berlin 1995)

[8] S.Brennan et P.Eisenberger, Nucl. Instr. and Meth. 222 (1984) 164

[9] P.H.Fuoss et I.K.Robinson, Nucl. Instr. and Meth. 222 (1984) 171

[10] E. Vlieg et coll. Nucl. Instr. and Meth. A262(1987) 522

[11] P.Claverie et coll. Rev. Sci. Instrum. 60 (1989) 2369

[12] G.Renaud, B.Vilette et P.Guénard, Nucl. Instr. and Meth. B95 (1995) 422 